\title{
Evaluation de l'état trophique des eaux du complexe lagunaire côtier du Sud- Ouest Bénin en période de hautes eaux (de Togbin à l'Embouchure Mono)
}

\author{
O. B. M. Lucinde BOCODAHO ${ }^{2 *}$, Waris Kéwouyèmi CHOUTI ${ }^{1,2}$, \\ Comlan Achille DEDJIHO ${ }^{1}$ et Daouda MAMA ${ }^{1,2}$ \\ ${ }^{1}$ Laboratoire d'Hydrologie Appliquée, Institut National de l'Eau (INE), Université d'Abomey-Calavi 01 \\ BP: 526 Cotonou, Bénin. \\ ${ }^{2}$ Laboratoire de Chimie Inorganique et de l'Environnement, Faculté des Sciences et Techniques (FAST), \\ Université d'Abomey-Calavi, BP : 4521 Cotonou, Bénin. \\ *Auteur correspondant ; E-mail : bokomarthe@yahoo.com ; Tél : +229 96130184
}

\section{RESUME}

Les lagunes servent de tanières pour un grand nombre d'espèces aquatiques et constituent de ce fait des richesses à entretenir. Malheureusement, la lagune côtière du Bénin fait face à un déséquilibre écologique qui se manifeste par une croissance excessive des végétaux aquatiques. En vue de caractériser l'état trophique de la lagune, une campagne d'échantillonnage a été effectuée en période de hautes eaux sur onze points représentatifs. La méthodologie de dosage au spectrophotomètre a été adoptée pour mettre en évidence les paramètres de diagnostic de pollution. Les analyses signalent de fortes concentrations en phosphore $(28,75 \mathrm{mg} / \mathrm{L})$ et en nitrites $(10 \mathrm{mg} / \mathrm{L})$ dans les eaux de la lagune. La lagune se trouve alors dans un état d'hyper-eutrophisation selon la grille de qualité établie par Ifremer. Le non-respect des mesures de protection des plans d'eau et la mauvaise gestion des déchets ménagers et des eaux usées entraînent la prolifération des nutriments responsables de l'eutrophisation de la lagune côtière. Vu l'ampleur de la pollution mise en relief par cette étude, l'institution d'un programme de surveillance s'avère indispensable afin de veiller au maintien de la qualité écologique des écosystèmes aquatiques en République du Bénin.

(C) 2020 International Formulae Group. All rights reserved.

Mots clés : Lagune côtière, eutrophisation, nutriments, pollution.

\section{Assessment of the trophic state of the waters of the coastal lagoon complex of Southwest Benin during high water periods (from Togbin to Mono Mouth)}

\begin{abstract}
The lagoons serve as places of residence for several aquatic species and therefore constitute riches to be properly maintained. Unfortunately, the coastal lagoon of Benin faces an ecological imbalance visibly manifested by an excessive growth of aquatic plants. In order to characterize the trophic state of the lagoon, a sampling campaign was carried out during high water periods according to eleven representative points. The spectrophotometer assay methodology was adopted to highlight the pollution diagnostic parameters. Analyzes report high concentrations of phosphorus $(28.75 \mathrm{mg} / \mathrm{L})$ and nitrites $(10 \mathrm{mg} / \mathrm{L})$ in lagoon waters. The lagoon is then in an enlarged state according to the quality grid established by Ifremer. Failure to comply with measures
\end{abstract}


to protect water bodies and poor management of household waste and wastewater lead to the proliferation of nutrients responsible for eutrophication within the coastal lagoon. Given the magnitude of the pollution highlighted by this study, the implementation of a monitoring program is essential in order to ensure the maintenance of the ecological quality of aquatic ecosystems in the Republic of Benin.

(C) 2020 International Formulae Group. All rights reserved.

Keywords: Coastal lagoon, eutrophication, nutrients, pollution.

\section{INTRODUCTION}

En Afrique de l'Ouest, le phénomène d'eutrophisation des plans d'eaux a connu une évolution ces dernières décennies avec la forte augmentation des rejets anthropiques en azote et en phosphore (Camus, 2014). Au Bénin, spécifiquement en zones côtières, les déchets solides ménagers tout comme les eaux usées domestiques et les eaux vannes sont déversés dans la nature ou les plans d'eaux sans aucun traitement préalable. En effet, ces déchets chargés de matières organiques et d'éléments nutritifs s'infiltrent directement ou indirectement dans les eaux de surface et perturbent leurs équilibres naturels visiblement manifestes par l'eutrophisation (Pinay et al., 2018). A l'instar du lac Nokoué (Mama, 2010), de la lagune de Porto-Novo (Mama et al., 2011), la lagune côtière située dans la région Sud-Ouest du Bénin est sujette au phénomène d'eutrophisation. Or les lagunes jouent un rôle capital pour le fonctionnement de la biodiversité et constituent une puissance économique de par leur forte potentialité de production en espèces halieutiques (Ruiz et al., 2006).

Malgré les travaux de différents auteurs sur la lagune côtière, plusieurs aspects demeurent encore non abordés. Le présent travail vise à évaluer la qualité physicochimique et l'état trophique des eaux de la lagune afin d'identifier les risques potentiels pour les espèces aquatiques et la santé humaine.

\section{MATERIEL ET METHODES \\ Milieu d'étude}

L'étude est menée sur la lagune côtière au Sud-Ouest du Bénin, en Afrique de l'Ouest. Elle fait partie du site Ramsar 1017 et appartient au complexe Ouest du Grand Bassin Sédimentaire Côtier (Baglo et al., 1982). Le système lagunaire s'étend sur $65 \mathrm{~km}$ de la frontière du Togo à Togbin (moins de $10 \mathrm{~km}$ de Cotonou) et se subdivise en lagune côtière de Ouidah et de Grand-Popo de part et d'autres du chenal Aho (Guilcher, 1959). Dans son bassin versant, se développent l'agriculture, la fabrication du sel, l'exploitation de la palmeraie et la pêche qui se pratique avec la méthode abusive d'acadjas. La lagune côtière se caractérise par des apports d'eau salée issus de la mer, des apports d'eaux douces provenant du fleuve Mono et du fleuve Couffo (Chitou, 2017).

\section{Prélèvement et méthodes d'analyse}

Les données de cette étude proviennent de onze (11) stations d'échantillonnage dont le motif de leurs choix et les coordonnées géographiques sont présentés dans le Tableau 1. Une succession de deux (2) campagnes d'échantillonnage a été effectuée sur la lagune côtière en période de hautes eaux, afin de suivre la variation spatiale des paramètres physico-chimiques des eaux de la lagune. Les échantillons d'eaux ont été prélevés dans des bouteilles en plastiques de $1,5 \mathrm{~L}$ à quelques centimètres de la surface de l'eau et sont conservés au frais dans un sac glacière portatif puis conduits au laboratoire pour des analyses chimiques.

Le Tableau 2 récapitule les différentes méthodologies adoptées selon chaque paramètre évalué.

La détermination de l'état trophique de la lagune a été basée sur la grille d'évaluation $\mathrm{du}$ risque d'eutrophisation selon Ifremer (2004). 
Tableau 1 : Les stations de prélèvement et motivation de leur choix.

\begin{tabular}{|c|c|c|c|}
\hline Sites & Noms & Motifs & Coordonnées (UTM) \\
\hline S1 & Togbin & $\begin{array}{l}\text { Production massif de déchets issus de plusieurs } \\
\text { agglomérations, des écoles et complexes hôteliers }\end{array}$ & $\begin{array}{l}423416 \\
702320\end{array}$ \\
\hline $\mathbf{S 2}$ & Hyо & $\begin{array}{l}\text { Déversement anarchique des déchets autour de la } \\
\text { lagune }\end{array}$ & $\begin{array}{l}416937 \\
701593\end{array}$ \\
\hline S3 & Vodounto & Site mise à part et protégé par les rituels endogènes & $\begin{array}{l}414844 \\
701689\end{array}$ \\
\hline S4 & Avlékété & $\begin{array}{l}\text { Présence des déchets solides ménagers et latrines } \\
\text { étanches aux abords de la lagune }\end{array}$ & $\begin{array}{l}412203 \\
701608\end{array}$ \\
\hline S5 & Agouin & $\begin{array}{l}\text { Village sur la berge : Mauvaises gestion des déchets } \\
\text { solides ménagers }\end{array}$ & $\begin{array}{l}408484 \\
700842\end{array}$ \\
\hline S6 & Djègbadji & $\begin{array}{l}\text { Réceptacle des eaux de ruissellement et extraction } \\
\text { artisanale du sel : voir les effets de ces méthodes } \\
\text { sur la lagune }\end{array}$ & $\begin{array}{l}399072 \\
699932\end{array}$ \\
\hline S7 & Azizakouè & $\begin{array}{l}\text { Élevage des porcs, et rejets d'ordures ménagères, } \\
\text { défécation des êtres humains sur la berge. }\end{array}$ & $\begin{array}{l}392918 \\
699061\end{array}$ \\
\hline S8 & Dondji & $\begin{array}{l}\text { Point de contact de la lagune avec le lac Ahémé : } \\
\text { évaluer les apports du lac Ahémé }\end{array}$ & $\begin{array}{l}386919 \\
697647\end{array}$ \\
\hline S9 & Embouchure & $\begin{array}{l}\text { Ouverture de la lagune sur l'océan atlantique : } \\
\text { examiner l'impact de la mer sur la lagune }\end{array}$ & $\begin{array}{l}381641 \\
696342\end{array}$ \\
\hline S10 & Avlo & $\begin{array}{l}\text { Point montrant la lagune à l'épicentre de deux } \\
\text { grands villages }\end{array}$ & $\begin{array}{l}377930 \\
695844\end{array}$ \\
\hline S11 & Entrée Mono & $\begin{array}{l}\text { Ouverture de la lagune sur le fleuve Mono : évaluer } \\
\text { les apports du fleuve Mono sur la lagune }\end{array}$ & $\begin{array}{l}372808 \\
694914\end{array}$ \\
\hline
\end{tabular}

Tableau 2 : Tableau récapitulatif de la méthodologie des paramètres évalués.

\begin{tabular}{|c|c|}
\hline Paramètres recherchés & Appareils et méthode \\
\hline $\begin{array}{l}\text { Température, Oxygène dissous, salinité, } \\
\text { conductivité, turbidité }\end{array}$ & $\begin{array}{l}\text { Mesure in situ par le conductimètre multi paramètre de } \\
\text { type WTW } 3630 \text { IDS }\end{array}$ \\
\hline $\mathrm{pH}$ & Mesure in situ par le pH-mètre WTW 3630 IDS \\
\hline Transparence & Mesure in situ au moyen du disque de Secchi \\
\hline Coordonnées géographiques & GPS GARMIN \\
\hline Matières en suspension (MES) & Filtration sur membrane \\
\hline Ions majeurs & $\begin{array}{l}\text { Méthode spectrophotométrique } \mathrm{HACH} \text { LANGE } \\
\text { DR2800 }\end{array}$ \\
\hline Phosphore Total & $\begin{array}{l}\text { Dosage au spectrophotomètre } \\
\text { moléculaire DR } 3900 \\
\text { Réactif : PhosVer®3 }\end{array}$ \\
\hline Nitrites & $\begin{array}{l}\text { Méthode : réduction au cadmium } \\
\text { Réactif : NitriVer®2 }\end{array}$ \\
\hline Nitrates & $\begin{array}{l}\text { Méthode : sulfate ferreux } \\
\text { Réactif : NitraVer@5 }\end{array}$ \\
\hline
\end{tabular}




\section{RESULTATS}

Les résultats issus des analyses physicochimiques des eaux de la lagune côtière sont traduits par les Figures 2 à 6 et ceux des ions en solutions sont récapitulés dans le Tableau 5 . La Figure 6 illustre les teneurs obtenues en azote et phosphore et les Tableaux 3 et 4 traduisent les grilles de l'état trophique de la lagune.

Les valeurs enregistrées pour la température varient entre 27,3 et $29,5{ }^{\circ} \mathrm{C}$. Quant au $\mathrm{pH}$, les mesures oscillent autour de 7,55 et 7,85 et sont conformes aux normes admises par l'OMS. Il ressort que les eaux de la lagune côtière sont légèrement alcalines. Le pic de la température a été observé à Togbin et celui du pH à Avlo.

La conductivité et le TDS présentent les mêmes allures sur toutes les stations et varient respectivement de 0,09 à $14,82 \mathrm{~ms} / \mathrm{cm}$ et de 0,09 à $14,82 \mathrm{~g} / \mathrm{L}$. La salinité oscille entre 0 à $8,6 \mathrm{~g} / \mathrm{L}$ et évolue de façon tendancielle avec la conductivité et la TDS. Les trois paramètres sont plus élevés à Djègbadji, Agouin et Avlékété et plus faibles à Avlo et Entrée Mono.

Les teneurs en oxygène dissous sont supérieures à $3 \mathrm{mg} / \mathrm{L}$ sur l'ensemble des stations. La transparence est pratiquement nulle sur toutes les stations, tandis que la turbidité et les matières en suspension ont présenté des valeurs élevées à hauteur de 41,6 NTU et $100 \mathrm{mg} / \mathrm{L}$ respectivement. Les eaux sont plus troubles au niveau des stations Djègbadji, Avlo et Embouchure tandis que les apports en MES s'observent à Avlo et à l'Entrée Mono. Les résultats obtenus mettent en exergue un lien étroit de causalité entre ces trois paramètres : la transparence diminue alors que la turbidité et les matières en suspension augmentent.

On assiste à un enrichissement de toutes les stations en phosphores, nitrites et nitrates signalant de forts pics à Avlékété, Djègbadji, Dondji et Avlo. Le phosphore présente des concentrations relativement élevées sur la quasi-totalité des stations variant entre 6,31 et $28,75 \mathrm{mg} / \mathrm{L}$ avec un minima à Agouin et un maxima à Avlékété et à l'Entrée Mono.

La caractérisation de l'état trophique de la lagune suivant les onze (11) points, sont traduits par les Tableaux 4 et 5 .

Les résultats issus du diagnostic de la grille de Ifremer, classent la turbidité dans la catégorie du « bon » au « moyen » sur $45 \%$ des sites et les $55 \%$ restants dans la catégorie de «médiocre » à «mauvais ». Les nitrites et nitrates se retrouvent majoritairement dans la mauvaise « classe », soit $73 \%$ des échantillons. Par contre, le phosphore total s'inscrit dans la «mauvaise » gamme sur la totalité des sites. L'écart moyen par rapport à la saturation en oxygène signale une sursaturation.

La dureté des eaux de la lagune est comprise entre 0,12 et $3,12 \mathrm{mg} / \mathrm{L}$ de $\mathrm{CaCO}_{3}$. Les ions chlorures ont une concentration variante entre 11,8 à $265,3 \mathrm{mg} / \mathrm{L}$. En ce qui concerne le potassium, neuf (9) sites sur les onze (11) présentent des teneurs très élevées excédant de loin les normes admises par l'OMS. Seule la station Dondji présente une pollution en ions chlorure.

Tableau 3 : Grille de diagnostic de l'état trophique des eaux de la lagune côtière.

\begin{tabular}{|c|c|c|c|c|c|c|c|c|c|c|}
\hline Variables & & $\begin{array}{l}\text { Très } \\
\text { bon }\end{array}$ & & Bon & & Moyen & & Médiocre & & Mauvais \\
\hline$\Delta(\%) \mathrm{O}_{2}$ sat & 0 & & 20 & & 30 & & 40 & & 50 & 63 \\
\hline TUR (NTU) & 0 & & 5 & 7,3 & 10 & 15,5 & 25 & 29 & 40 & 38 \\
\hline NITRA $(\mu \mathrm{M})$ & 0 & 0,16 & 1 & & 3 & & 5 & & 10 & 47,17 \\
\hline NITRI $(\mu \mathrm{M})$ & 0 & 0,22 & 0,3 & & 0,5 & & 0,75 & & 1 & 76,08 \\
\hline $\mathrm{PT}(\mu \mathrm{M})$ & 0 & & 0,75 & & 1,5 & & 2,5 & & 4,5 & 405,65 \\
\hline
\end{tabular}




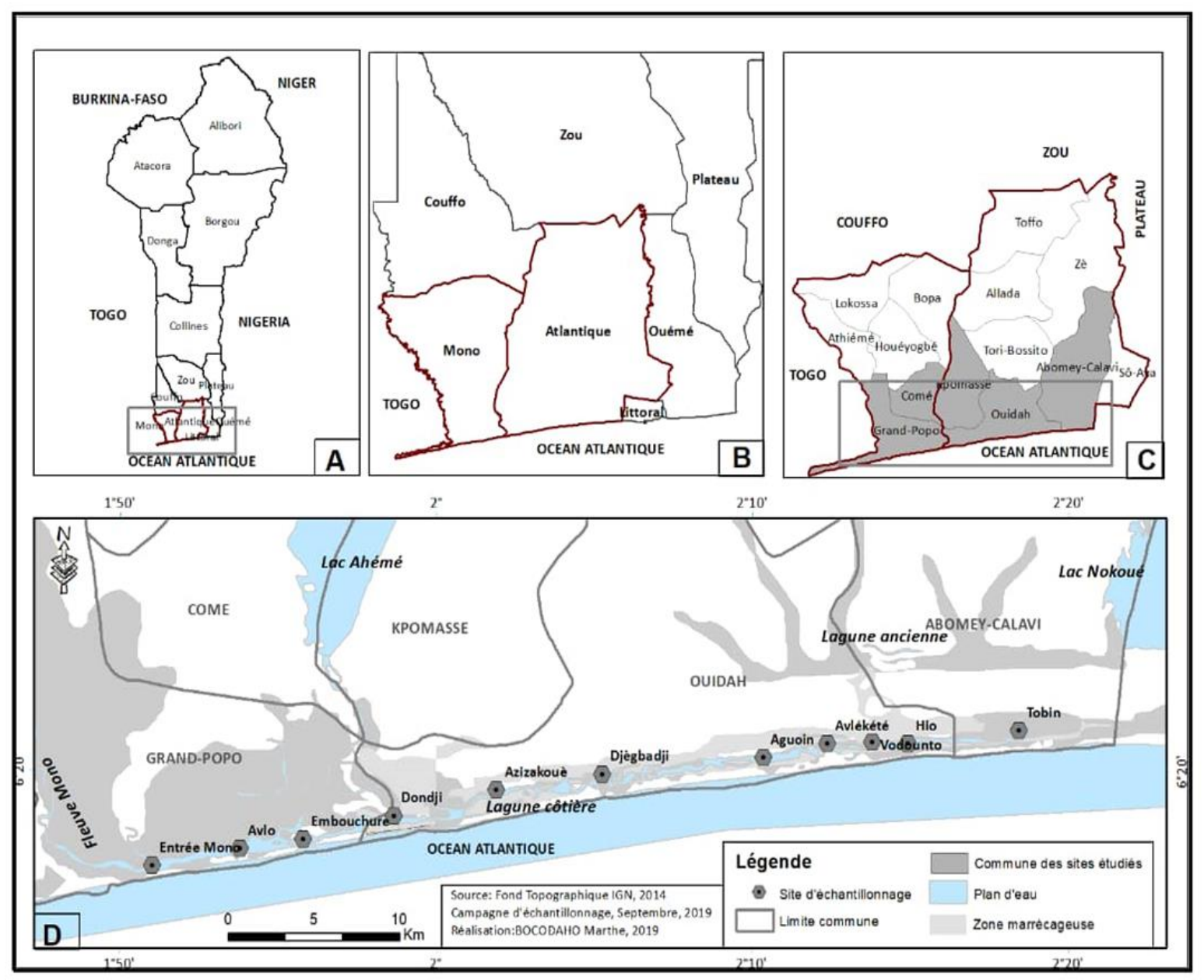

Figure 1 : Situation géographique de la zone d'étude et stations de prélèvement.

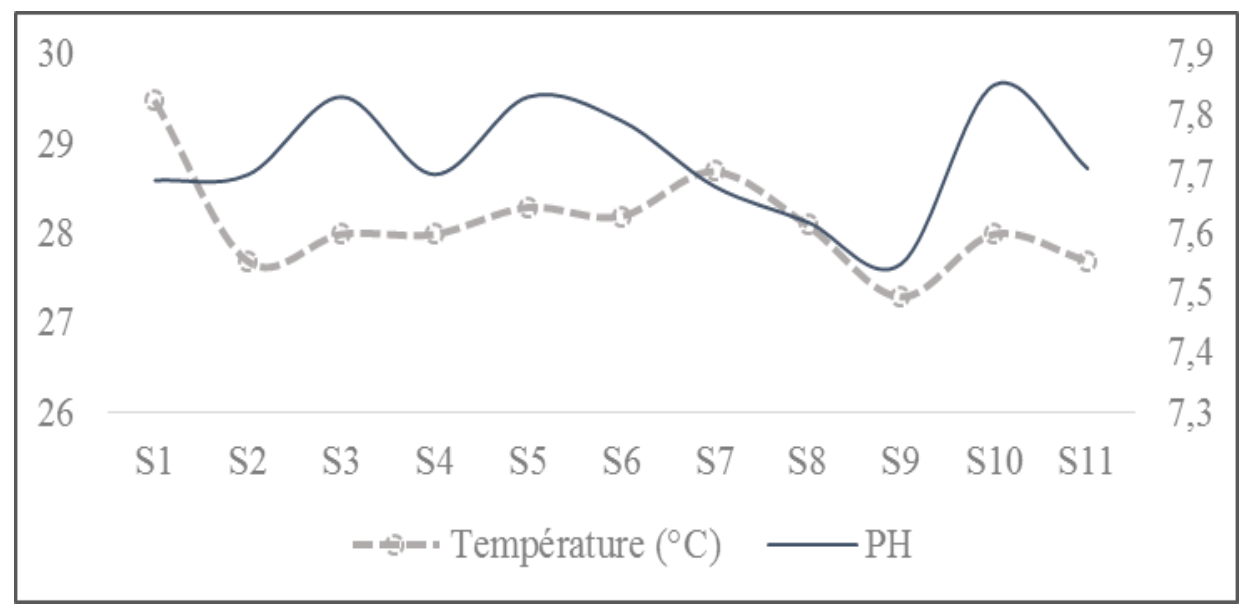

Figure 2 : Variation de la température et du pH suivant les sites d'échantillonnage. 


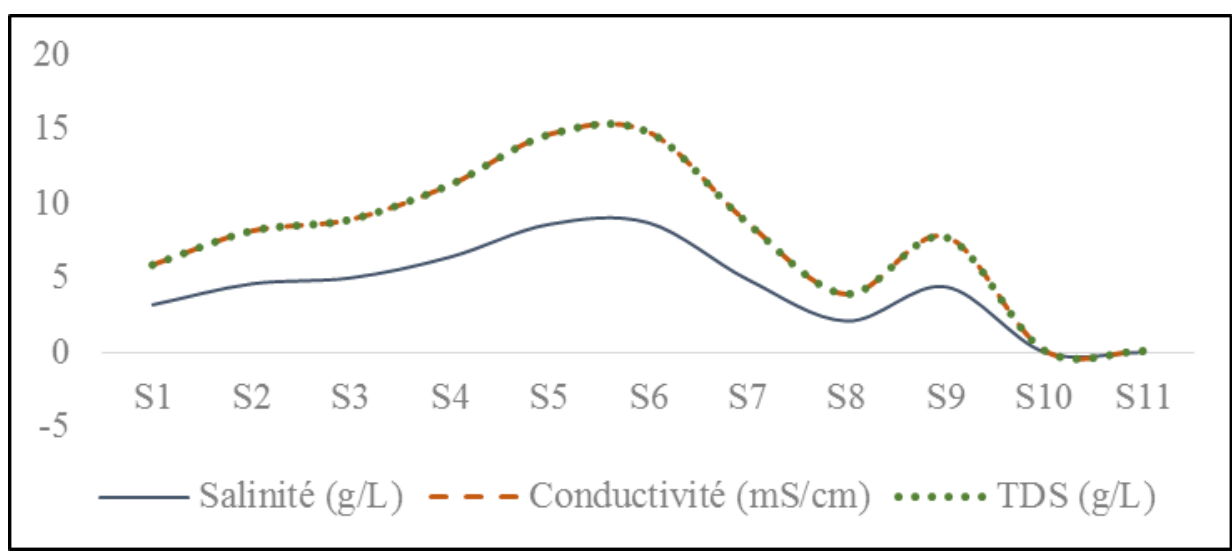

Figure 3 : Variation du TDS, conductivité et salinité suivant les sites d'échantillonnage.

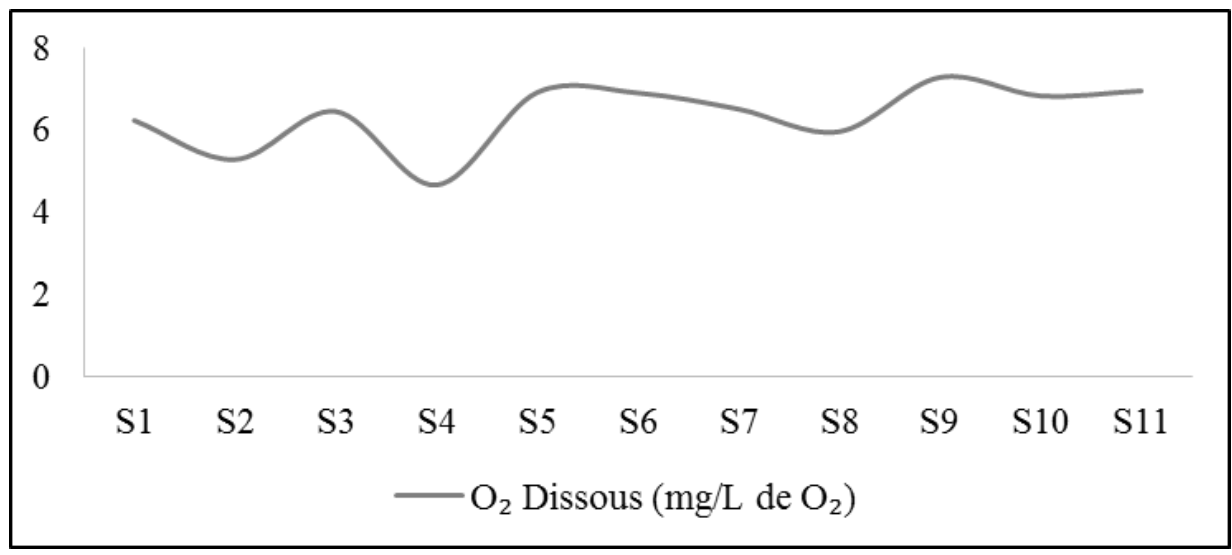

Figure 4 : Variation de l'oxygène dissous suivant les sites d'échantillonnage.

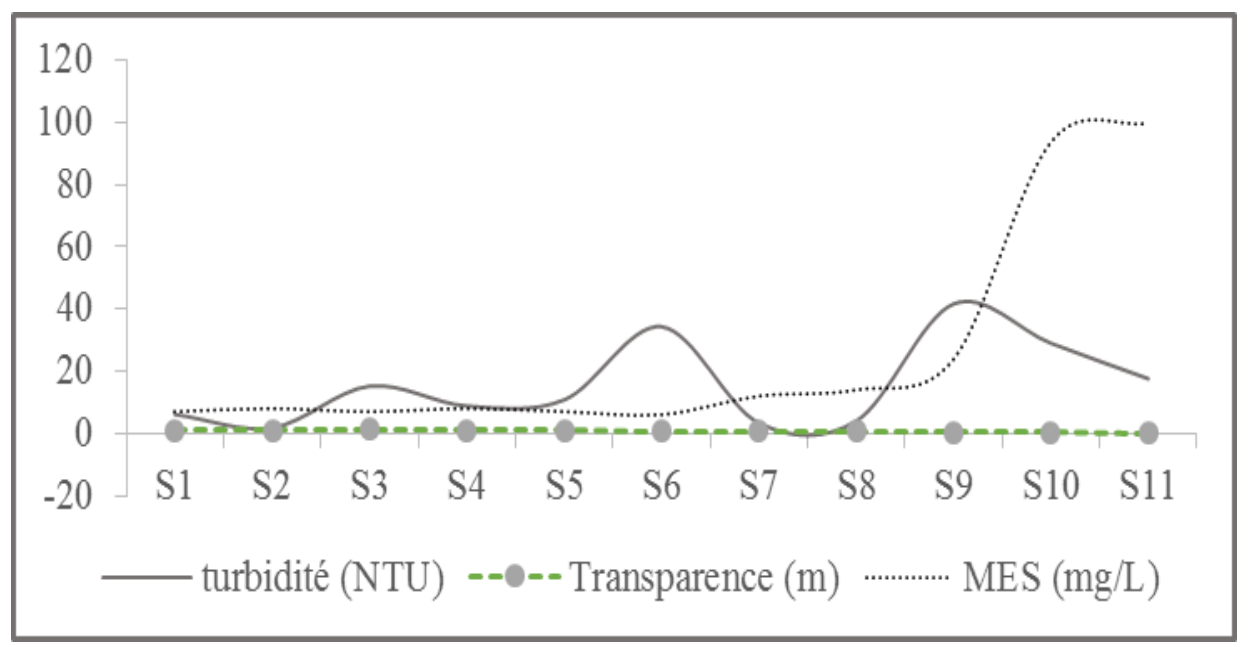

Figure 5 : Evolution de la turbidité, transparence et matières en suspension selon les stations. 


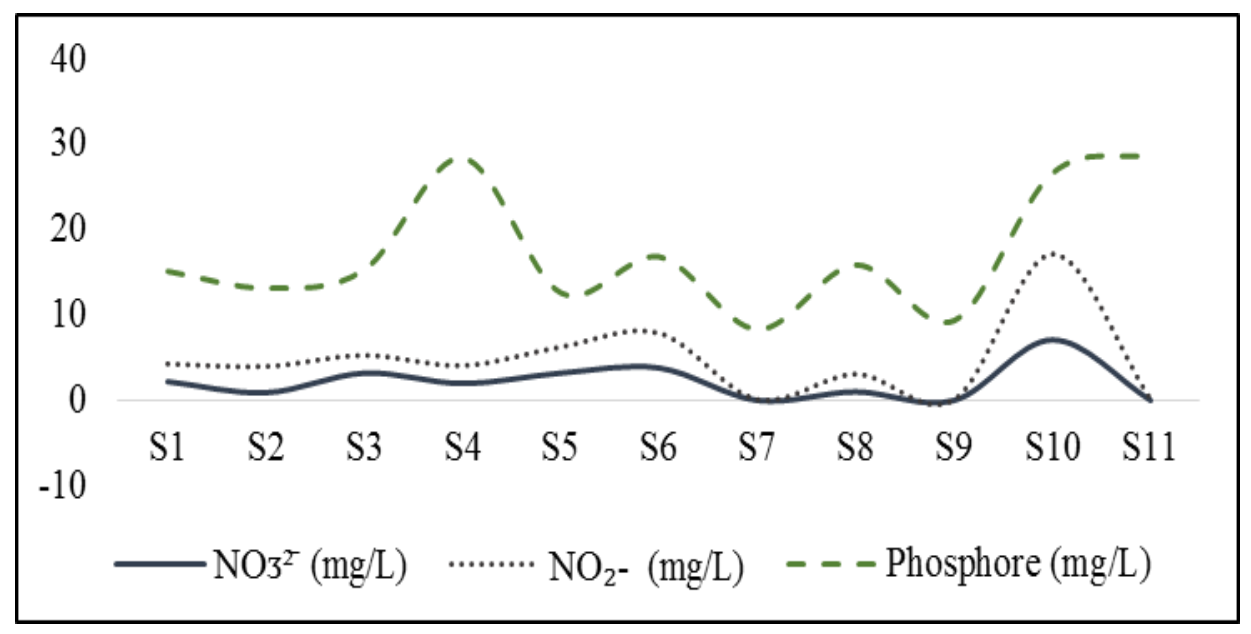

Figure 6 : Variation du phosphore et de l'azote suivant les sites d'échantillonnage.

Tableau 4 : Classification de la qualité des eaux de la lagune côtière.

\begin{tabular}{|c|c|c|c|c|}
\hline Variables & Oligotrophie & Mésotrophie & Eutrophie & Hypertrophie \\
\hline Turbidité & $\begin{array}{l}3 \text { cas : } \\
\text { S2, S7, S8 }\end{array}$ & 2 cas : $\mathrm{S} 1, \mathrm{~S} 4$ & $\begin{array}{l}3 \text { cas: } S 4, \text { S6, } \\
\text { S11 }\end{array}$ & 3 cas : $S 5, S 8, S 9$ \\
\hline Nitrates & $\begin{array}{l}3 \text { cas : } \\
\text { S7, S9, S11 }\end{array}$ & Néant & Néant & 8 sites \\
\hline Nitrites & $\begin{array}{l}3 \text { cas : } \\
\text { S7, S9, S11 }\end{array}$ & Néant & Néant & 8 sites \\
\hline Phosphore T & Néant & Néant & Néant & 11 sites \\
\hline$\Delta(\%) O_{2}$ sat & Néant & Néant & 1 site & 10 sites \\
\hline
\end{tabular}

Tableau 5 : Tableau récapitulatif des concentrations relatives $(\mathrm{mg} / \mathrm{L})$ des ions majeurs dans les eaux de la lagune.

\begin{tabular}{ccccccc}
\hline Sites & $\mathbf{C a}^{\mathbf{2 +}}$ & $\mathbf{M g}^{\mathbf{2 +}}$ & $\mathbf{K}^{+}$ & $\mathbf{F}^{-}$ & $\mathbf{C l}^{-}$ & ${\text {Dureté }\left({ }^{\circ} \mathbf{f}\right)}^{\mathbf{S}}$ \\
$\mathbf{S 1}$ & 0,08 & 0,04 & 167 & 1,05 & 163,6 & 1,2 \\
S2 & 0,22 & 0,07 & 160,7 & 1,21 & 120,6 & 2,9 \\
S3 & 0,32 & 0,09 & 193,9 & 0,95 & 131,3 & 4,1 \\
S4 & 0,11 & 0,01 & 205,2 & 1,2 & 125,5 & 1,2 \\
S5 & 0,12 & 0,06 & 168,3 & 1,28 & 76,4 & 1,8 \\
S6 & 0,35 & 0,08 & 179,8 & 1,06 & 158,2 & 4,3 \\
S7 & 0,17 & 0,04 & 144,1 & 0,8 & 109,1 & 2,1 \\
S8 & 0,77 & 0,26 & 69,9 & 0,73 & 256,3 & 10,3 \\
S9 & 0,12 & 0,02 & 111,1 & 0,58 & 164,7 & 1,4 \\
S10 & 2,45 & 0,67 & 2,4 & $<0,01$ & 6,1 & 31,2 \\
\hline S11 & 2,28 & 0,66 & 2,7 & $<00,1$ & 11,8 & 29,4 \\
\hline
\end{tabular}




\section{DISCUSSION}

Les résultats relatifs aux paramètres physico-chimiques et chimiques illustrés ont permis d'apprécier le phénomène d'eutrophisation au sein de la lagune côtière du Bénin. Les valeurs moyennes de $28{ }^{\circ} \mathrm{C}$ pour la température enregistrées au niveau des stations sont similaires à celles des études précédentes de Chitou en 2017 sur la même lagune. Ces valeurs correspondent également aux températures observées par Dèdjiho (2011) et Chouti (2011) respectivement dans les eaux du lac Ahémé et la lagune de Porto-Novo. Or, Baras et Jobling (2002) conclurent qu'une température de l'ordre de $28{ }^{\circ} \mathrm{C}$ favorise une bonne croissance du poisson-chat. De même, Abou (2001) réalise qu'une température située entre 28 et $32{ }^{\circ} \mathrm{C}$ est idéale pour la croissance des tilapias. Par ailleurs, les valeurs de $\mathrm{pH}$ obtenues sont bonnes en aquaculture car situées entre 6,5 à 9 (Kanangire, 2001). Ces valeurs sont conformes aux travaux de Falconbridge (2001) qui conclut qu'une eau à $\mathrm{pH}$ naturel d'intervalles 6,5 et 9,5 est un bon indicateur pour le développement optimal du milieu aquatique. Donc, la température et le $\mathrm{pH}$ des eaux de la lagune côtière du Sud-Ouest Bénin, répondent aux normes admises pour la protection de la vie des espèces halieutiques. La conductivité et les TDS sont en forte corrélation et sont liées à la nature et à la concentration de substances dissoutes du milieu. D'après les résultats, environ $82 \%$ des points échantillonnés sont largement supérieures à la norme admise pour les eaux naturelles $(0,05$ à $1,5 \mathrm{mS} / \mathrm{cm})$. Les eaux de la lagune côtière présentent alors une forte minéralisation des sels présents dans le milieu. La forte teneur en salinité observée à Djègbadji pourrait s'expliquer par l'extraction du sel qui est la principale activité de commercialisation dans ce village. L'oxygène dissout présente des valeurs excédant les normes requises $(6 \mathrm{mg} / \mathrm{L})$ sur huit stations. Soit plus de $72 \%$ des points échantillonnés. La valeur maximale $(7,3 \mathrm{mg} / \mathrm{L})$ obtenue signale une eutrophisation du milieu qui se traduit par une photosynthèse intense. Les fortes fluctuations des matières en suspension et de la turbidité sont responsables de la baisse de la transparence. Les valeurs observées pour la transparence sont toutes inférieures à $1,5 \mathrm{~m}$ sur toutes les stations. En se référant donc à l'Organisation de Coopération et Développement Economique (OCDE) sur cette base, la lagune côtière se retrouve dans la catégorie des plans d'eaux hyper-eutrophes.

En ce qui concerne les nitrites, nitrates et phosphores, leurs teneurs sont excessivement élevées dans les eaux de la lagune avec des valeurs excédantes les normes recommandées sur $73 \%$ environ des échantillons. Au total, huit (8) stations sur les onze (11) présentent des valeurs élevées pour le couple (nitrites/nitrates) oscillant autour de 3 et $17 \mathrm{mg} / \mathrm{L}$ avec une moyenne de $3 \mathrm{mg} / \mathrm{L}$ pour le nitrate et $3,5 \mathrm{mg} / \mathrm{L}$ pour le nitrite. Par contre, au niveau de trois autres stations, les teneurs sont pratiquement en traces. La station Avlo se révèle la plus polluée de toutes en nitrates et en nitrites. Le dosage du phosphore et de l'azote dans les eaux, signale la présence de nutriments dans la lagune. Le fait que ces trois variables affichent des teneurs élevées sur l'ensemble des stations, traduisent une forte anthropisation probablement due aux déjections animales, aux urines et aux eaux usées domestiques qui sont déversées permanemment dans la lagune. En effet, Adjahouinou et al. (2002), démontra que les eaux usées domestiques tout comme les urines sont chargées de substances très riches en phosphores. Par ailleurs, ces apports externes d'azote et de phosphore, exclusivement issus des rejets d'effluents (domestiques, industriels et agricoles), accélèrent l'eutrophisation et entrainent le vieillissement des plans d'eaux par lixiviation d'eaux de ruissellement contaminées (Dovonou et al., 2011). Selon Trinquier (2009), l'eutrophisation se présente naturellement comme un phénomène géologiquement lent. Son amplification au sein de la lagune côtière, serait probablement le résultat malheureux des activités humaines dans la zone côtière. En conséquence, elle se manifeste surtout par une prolifération excessive d'algues qui appauvrit l'eau en oxygène occasionnant un risque accru de mortalité chez certains organismes aquatiques. La toxicité des nitrites sur les poissons varie selon les espèces. Cependant les nitrites sont surtout nuisibles pour les jeunes poissons (Zinsou et al., 2016). Pour Lisec (2004), la situation devient très critique au-delà 
d'une concentration de $3 \mathrm{mg} \mathrm{NO}_{2}^{-} / \mathrm{L}$. En revanche, selon Gray et al. (2002), des valeurs approximatives de $0,015 \mathrm{mg} / \mathrm{L}$ de nitrites causent la mort de certains poissons par hypoxie. Par ailleurs, Djibril en 2001 stipule qu'à partir d'une teneur de $0,06 \mathrm{mg} / \mathrm{L}$, les nitrites bloquent le développement de la faune aquatique. Les teneurs en nitrites sont principalement élevées au niveau des villes qui regorgent la majorité de la population, où la collecte et la gestion des déchets demeurent un épineux problème environnemental menaçant la sécurité sanitaire. La mauvaise gestion des ordures ménagères et des eaux usées au sein de ces villes provoque une forte pollution anthropique sur l'ensemble des ressources vitales, notamment l'eau. Les résultats obtenus allant jusqu'à $10 \mathrm{mg} / \mathrm{L}$ de nitrites, doivent faire l'objet d'une alerte forte à l'égard de toutes les couches concernées par la lagune côtière. La situation est remarquable à Hyo et Aguoin mais semble encore très critique à Avlo et Djègbadji. En effet, au niveau du site Avlo, la lagune est entourée par deux importants villages déversant leurs déchets chargés de polluants organique et minéral à la périphérie et autour de la lagune. Le site de Djègbadji de par son statut de zone de production et de commercialisation du sel, est un point culminant de réceptacle des eaux de ruissellement fortement chargées de déchets solides ménagers et des intrants agricoles provenant de part et d'autres de la lagune. Selon Dianou (2011), les fortes teneurs en nitrates s'observent généralement au niveau des sites de déjections animales, d'ordures ménagères et des eaux usées. Ces multiples raisons justifient les valeurs maximales de nitrites et de nitrates observées en ces points. En ce qui concerne le phosphore, une concentration excédant $0,5 \mathrm{mg} / \mathrm{L}$ constitue un risque de pollution pour les eaux (Ifremer, 2001). Les taux alarmants du phosphore présent dans la lagune, avec des valeurs au moins douze (12) fois supérieures à $0,5 \mathrm{mg} / \mathrm{L}$ au niveau de toutes les onze stations, expliquent le lessivage des terres agricoles dans la zone d'étude. La lagune côtière est alors très polluée par le phosphore. En outre, Dovonou et al. (2011), attribuent l'accumulation des engrais dans les terres agricoles en milieu rural comme étant la cause probable de l'excès du phosphore dans les cours d'eaux. Ces résultats sont similaires à ceux de Chouti et al. (2017) dans les eaux de la lagune de Porto-Novo, et Mama (2010) dans celles du lac Nokoué. Mais par contre, Zinsou et al. (2016), dans le Delta de l'Ouémé ont obtenu des teneurs relativement faibles en nitrites $(0,004$ à $0,13 \mathrm{mg} / \mathrm{L})$ et en nitrates $(0,5$ $\mathrm{mg} / \mathrm{L}$ ) sur l'ensemble des stations à l'exception de trois stations provenant directement des sites de réceptacles de déchets. Ansa-Asare et al. (2000), ainsi que Souaad et Belkheir. (2012), pensent que les activités humaines engendrent une forte concentration de nutriments notamment l'azote et le phosphore. Du dosage des ions calcium et magnésium, les eaux de la lagune côtière sont majoritairement douces à l'exception des eaux des sites Avlo et Entrée Mono qui sont dures. Le fluor paraît en concentration élevée à la limite $(0,7 \mathrm{mg} / \mathrm{L})$ sur $64 \%$ environ des échantillons alors que les ions chlorures sont conformes aux normes à l'exception d'une seule station qui dépasse légèrement la norme OMS. La concentration élevée en ions chlorures à Dondji pourrait indiquer une pollution par des eaux usées domestiques. Les concentrations en potassium excédant largement sur plus de $80 \%$ des échantillons, signale l'utilisation d'engrais dans la périphérie de la lagune. L'évaluation du risque d'eutrophisation par rapport à l'outil de diagnostic d'Ifremer (2004) révèle des valeurs, quatre (4), dix (10) et quatre-vingt-dix (90) fois supérieures aux valeurs de la grille de mauvais état pour le nitrate, le nitrite et le phosphore. La procédure de détermination de l'état trophique d'Ifremer, qualifie donc la lagune côtière d'hyper-eutrophe et la classe en zones à risque.

\section{Conclusion}

$\mathrm{Au}$ Bénin, le phénomène d'eutrophisation des ressources en eau est d'actualité. Cette étude a mis en relief la vulnérabilité de la lagune côtière face à l'eutrophisation et l'évolution des paramètres physico-chimiques en hautes eaux. Les mauvaises pratiques humaines face au nonrespect des normes de rejets des déchets ménagers et des eaux usées modifient sans doute la qualité et la dynamique de la lagune 
côtière. A cela s'ajoutent l'utilisation abusive d'intrants agricoles chargés d'azotes et de phosphores, principaux responsables de l'eutrophisation de la lagune. La politique de gestion des déchets et la réduction des engrais chimiques doivent être revues au niveau de ce plan d'eau afin de préserver cette importante ressource contre les conséquences drastiques de l'eutrophisation.

\section{CONFLIT D'INTERETS}

Les auteurs signalent que cet article ne présente aucun conflit d'intérêts.

\section{CONTRIBUTIONS DES AUTEURS}

OBMLB a élaboré le protocole et rédigé l'article. WKC et DM ont coordonné la rédaction et participé à la planification de l'étude et CAD a orienté la révision du document et contribué au financement. Tous les auteurs ont lu et approuvé le manuscrit final.

\section{REMERCIEMENTS}

Les auteurs tiennent à remercier $\mathrm{Mr}$ Michaël TOSSOU pour sa franche collaboration dans la réalisation des travaux de terrain.

\section{REFERENCES}

Abou Y. 2001. Effet d'une couverture d'Azolla LAM sur la production piscicole du Tilapia Oreochromisniloticus (L.) en étangs dans la banlieue de Cotonou (Bénin). Mémoire présenté pour l'obtention du diplôme d'études complémentaire en éco-technologie des eaux continentales. Facultés Universitaires Notre Dame de la Paix. Faculté des Sciences. Namur-Belgique. $85 \mathrm{p}$.

Adjahouinou DC, Liady ND, Fiogbé ED. 2012. Diversité phytoplanctonique et niveau de pollution des eaux du collecteur de Dantokpa (Cotonou-Bénin). Int. J. Biol. Chem. Sci., 6(5): 1938-1949. DOI : https://dx.doi.org/10.4314/ijbcs.v6i5.4.

Ansa-Ansare OD, Marr IL, Cresser MS. 2000. Evaluation of modeled and measured pattems of dissolved oxygen in a freshwater lake as an indicator of the presence of biodegradable organique pollution. Water Research, 34(4): 10791088.

DOI : https://doi.org/10.1016/S0043-1354(99) 00239-0.

Baglo AM, Thexier H. 1982. Développement de la production du sel marin en RPB. Groupe multidisciplinaire de recherche scientifique. «Lagunes et Mangroves », UNB, Cotonou, Bénin $36 \mathrm{p}$.

Baras E, Jobling M. 2002. Dynamics of intracohort cannibalism in cultured fish. Aquaculture Research, 3(7): 461-479. DOI : $\quad$ https://doi.org/10.1046/j.13652109.2002.00732.x

Camus G. 2014. Eutrophisation des eaux continentales de surfaces et marines Planet-Vie : $\quad$ https://planetvie.ens.fr/article/2095/eutrophisation

Chitou A. 2017. Caractérisation physicochimique et toxicité d'une lagune tropicale : cas de la lagune côtière (de Togbin à l'Embouchure) du Sud-Ouest du Benin. Mémoire de DEA, 73p.

Chouti WK, Atchichoe W, Tometin L, Mama D. 2017. Biodisponibilité et mobilité du phosphore des sédiments de la lagune de Porto-Novo. Journal of Applied Biosciences, 114: 11273-11288. DOI : https://dx.doi.org/10.4313/jab.v114i1.1

Chouti W. 2011. Etude de la pollution chimique d'une lagune tropicale (eaux, sédiments, poissons) : Cas de la lagune de Porto-Novo (Sud Bénin). Thèse de doctorat à l'Université d'Abomey-Calavi, $100 \mathrm{p}+$ Annexes.

Dianou D, Savadogo B, Zongo D, Zougouri T, Poda JN, Bado H, Rosillon F. 2011. Qualité des eaux de surface dans la vallée du Sourou: cas des rivières Mouhoun, Sourou, Debe et Gana au Burkina Faso. International Journal of Biological and Chemical Sciences, 5(4): 1571-1589. DOI :

http://dx.doi.org/10.4314/ijbcs.v5i4.22

Dèdjiho CA, 2011. Évaluation de la chaine trophique d'une aire marine protégée en relation avec sa physico-chimie : cas de Gbèzoumè dans la commune de Ouidah. Mémoire de DEA. FAST/UAC, Bénin. 
Djibril R, 2001. Impact de l'utilisation des engrais chimiques et des pesticides sur la qualité de l'eau de surface dans la réserve de la biosphère de la Pendjari. Mémoire de fin de formation CPU/UAC, 160p.

Dovonou F, Aina M, Boukari M, Alassane A, 2011. Pollution physico-chimique et bactériologique d'un écosystème aquatique et ses risques écotoxycologiques : cas du lac Nokoué du Sud Bénin. Int. J. Biol. Chem. Sci., 5(4): 1590-1602. DOI : 10.4314/ijbcs.v5i4.23.

Falconbridge NS. 2001. Etude environnementale sur la qualité de l'eau et des sédiments en milieu continental. Projet Koniambo.

Gray JS, Wu RSS, Or YY. 2002. Effects of hypoxia and organic enrichment of the coastal marine environment. Marine Ecology Progress Series, 238:249-279. DOI : 10.3354/meps238249.

Guilcher A, 1959. La région côtière du BasDahomey Occidental. Bull. de BIFAN, Série B, XXI (1-6): 358-423.

Ifremer. 2001. Suivi des paramètres trophiques dans l'eau des étangs de la Narbonnaise. 52p. https://archimer.fr

Ifremer. 2004. Direction de l'Environnement et de l'Aménagement Littoral, Laboratoire Environnement- Ressources du Languedoc-Roussillon, Rapport du Réseau Lagunaire du LanguedocRoussillon, Bilan des résultats 2003.

Kanangire CK. 2001. Effet de l'alimentaire des poisons avec Azolla sur l'écosystème agro-piscicole au Rwanda. Dissertation présentée en vue de l'obtention du grade de Docteur en sciences. Faculté Universitaire Notre Dame de la Paix. Faculté des sciences. Namur-Belgique. 220 p.

Lisec. 2004. Contrôle van de fysicochemische kwaliteit van de viswaters van het Brussels Hoofdstedelijk Gewest. Rapport effectué pour le compte de l'IBGE.

Mama D, Chouti W, Alassane A. Changotade O, Alapini F, Boukari M. 2011. Etude dynamique des apports en éléments majeurs et nutritifs des eaux de la lagune de Porto-Novo (Sud Bénin). Int. J. Biol. Chem. Sci., 5(3): 1278-1293. DOI : 10.4314/ijbcs.v5i3.72279.

Mama D. 2010. Méthodologie et résultats du diagnostic de l'eutrophisation du lac Nokoué (Bénin). Université de Limoges. Thèse de doctorat. 157p.

OCDE. 1982. Eutrophisation ses eaux : Méthodes de surveillance, d'évaluation et de lutte. Document OCDE. Paris : 1-65.

Pinay G, Gascuel C, Ménesguen A, Souchon Y, Le Moal M, Levain A, Etrillard C, Moatar F, Pannard A, Souchu P. 2018. L'eutrophisation : manifestations, causes, conséquences et prédictibilité. Editions Quae (France), 176 pages. www.cnrs.fr/inee.

Ruiz F, Abad M, Galan E, Gonzalez I, Aguila E, Olias M, Cantano M. 2006. The present environmentals scenario of the Nadoor Lagoon (Moroco). Environ. Res., 102: 215-229. DOI : 10.1016/j.envres.2006.03.001.

Souaad B, Belkheir D. 2012. Etude du phénomène d'eutrophisation dans le Barrage de Hammam Boughrara (Wilaya de Tlemcen, Algérie). Hydrological Sciences Journal, 5(1): 186-201. DOI : https://doi.org/10.1080/02626667.2011.6 34417.

Trinquier C. 2009. Le risque d'eutrophisation des lagunes Méditerranéennes : le cas de la lagune de Thau (Hérault). Master1 professionnel Gestion des catastrophes et des risques naturels. Université Paul Valéry-Montpellier III UFR III : Sciences Humaines et Sciences de l'Environnement. Département de Géographie. Aménagement 121p.

Zinsou HL, Attingli AH, Gnohossou P, Adandedjan D, Laleye P, 2016. Caractéristiques physico-chimiques et pollution de l'eau du delta de l'Ouémé au Bénin. Journal of Applied Biosciences, 97: 9163-9173. DOI : http://dx.doi.org/10.4314/jab.v97i1.3. 